\title{
Biofilm Formation and $\beta$-Lactamase Production in Burn Isolates of Pseudomonas aeruginosa
}

\author{
Samira Heydari ${ }^{1}$; Fereshteh Eftekhar ${ }^{1, *}$ \\ ${ }^{1}$ Department of Microbiology, Faculty of Biological Sciences, Shahid Beheshti University, Tehran, IR Iran \\ ${ }^{*}$ Corresponding author: Fereshteh Eftekhar, Department of Microbiology, Faculty of Biological Sciences, Shahid Beheshti University, Tehran, IR Iran. Tel: +98-2129903208, E-mail: \\ f-eftekhar@sbu.ac.ir
}

Received: October 20, 2013; Revised: February 19, 2014; Accepted: March 9, 2014

\begin{abstract}
Background: Pseudomonas aeruginosa is an important nosocomial pathogen characterized by its innate resistance to multiple antimicrobial agents. Plasmid-mediated drug resistance also occurs by the production of extended-spectrum $\beta$-lactamases (ESBL), metallo $\beta$-lactamases (MBL), and AmpC $\beta$-lactamases. Another important factor for establishment of chronic infections by P. aeruginosa is biofilm formation mediated by the psl gene cluster.

Objectives:The aim of this study was to evaluate biofilm formation and presence of the pslAgene in burn isolates of P. aeruginosa as well as the association of antibiotic resistance, MBL, ESBL and AmpC $\beta$-lactamase production with biofilm formation among the isolates.

Materials and Methods: Sixty-two burn isolates of P. aeruginos a were obtained from Shahid Motahari Hospital in Tehran from August to October 2011. Antibiotic susceptibility was determined by the disc diffusion assay. MBL, AmpC and ESBL production were screened using the double disc synergy test, AmpC disc test and combined disc diffusion assay, respectively. The potential to form biofilm was measured using the microtiter plate assay and pslA gene was detected using specific primers and PCR.

Results: Biofilm formation was observed in $43.5 \%$ of the isolates, of which $66.7 \%$ produced strong and $33.3 \%$ formed weak biofilms. All biofilm-positive and $14.2 \%$ of biofilm-negative isolates harbored the pslA gene. MBL, AmpC and ESBL production were significantly higher in the biofilm-positive isolates (70.3\%, 62.9\% and 33.3\%, respectively) compared to the biofilm-negative strains (31.4\%, 34.2\% and $20 \%$, respectively). Overall, 19 isolates (30.6\%) co-produced MBL and AmpC, among which the majority were biofilm-positive (63.1\%). Finally, four isolates (6.4\%) had all three enzymes, of which 3(75\%) produced biofilm.

Conclusions: Biofilm formation (both strong and weak) strongly correlated with pslA gene carriage. Biofilm formation also correlated with MBL and AmpC $\beta$-lactamase production. More importantly, multiple- $\beta$-lactamase phenotype was associated with formation of strong biofilms.
\end{abstract}

Keywords: Pseudomonas aeruginosa; $\beta$-Lactamase; biofilm; PslA

\section{Background}

Pseudomonas aeruginosa is an important opportunistic pathogen in nosocomial infections and responsible for high mortality rates in burn centers $(1,2)$. Infections caused by $P$. aeruginosa are difficult to treat, as the majority of isolates exhibit innate resistance to several antibiotics, due to poor outer-membrane permeability, constitutive expression of various efflux pumps and production of antibiotic inactivating enzymes (3). Among these, the important roles of various $\beta$-lactamases such as AmpC, extended-spectrum $\beta$-lactamases (ESBL) and carbapenemases have been reported (4-6). AmpC is responsible for resistance to cephalosporins and ESBLs confer resistance to all $\beta$-lactams except for the carbapenem family. Carbapenemases, particularly metallo $\beta$-lactamases (MBL), hydrolyze all $\beta$-lactam antibiotics with the exception of monobactams. Coexistence of multiple $\beta$-lactamases in clinical isolates of $P$. aeruginosa is common, causing resistance to almost all $\beta$-lactam antibiotics (7). Another important factor contributing to the pathogen- esis of $P$. aeruginosa in causing fatal infections is its potential to form biofilms on biotic and abiotic surfaces (8). The bacterial populations in biofilms are usually more resistant to antibiotics and host-mediated clearance strategies compared to their planktonic counterparts, giving rise to chronic infections that are notoriously difficult to eradicate $(9,10)$. Bacteria growing in biofilms produce one or more extracellular polymeric matrices which hold the cells of the biofilm community together. Polysaccharides are important components of the biofilm matrix, as they contribute to the overall biofilm architecture and to the resistance of biofilm-grown bacteria to certain antibacterial agents (11).

At least three exopolysaccharides have been shown to be involved in biofilm formation by P. aeruginosa, including alginate, Psl, and Pel (12). Among these, Psl is a mannoserich polymer with an essential role in the initial steps of biofilm formation by non-mucoid $P$. aeruginosa as well as in its maintenance. Psl forms a helical structure around

Copyright (C) 2015, Ahvaz Jundishapur University of Medical Sciences. This is an open-access article distributed under the terms of the Creative Commons Attribution-NonCommercial 4.0 International License (http://creativecommons.org/licenses/by-nc/4.0/) which permits copy and redistribute the material just in noncommercial usages, provided the original work is properly cited. 
Heydari S et al.

P. aeruginosa cells which increases the cell-to-surface and cell-to-cell interactions necessary for biofilm formation $(13,14)$. Synthesis of Psl is mediated by the psl gene cluster (pslA-pslO) and pslA has been reported to be the first and most important gene necessary for Psl synthesis $(15,16)$.

\section{Objectives}

Due to the suggested role of pslA in the initial steps of biofilm formation by $P$. aeruginosa, we studied the association between biofilm formation and the pslA gene carriage in burn isolates of $P$. aeruginosa. We also evaluated the potential to form biofilm in relation to antibiotic resistance and the production of AmpC, MBL and ESBL $\beta$-lactamases among the isolates.

\section{Materials and Methods}

\subsection{Bacterial Isolates}

Sixty-two isolates of $P$. aeruginosa were collected from hospitalized burned patients in Shahid Motahari Hospital, Iran, from August to October 2011 (17). The isolates were maintained in brain-heart infusion broth (Becton Dickinson, Franklin Lakes, NJ) containing 10\% dimethyl sulfoxide (DMSO) at $-20^{\circ} \mathrm{C}$ until use. The antibiotic susceptibility of the isolates had been previously determined for 13 antibiotics by the disc diffusion method (17). Staphy lococcus epidermidis strains RP62A and RP62NA were used as positive and negative controls for biofilm production, respectively (18). P. aeruginosa PAO1 was used as the positive control for pslA gene amplification.

\subsection{Biofilm Assay}

Biofilm formation was determined by the microtiter plate assay, as previously reported (18). Briefly, $200 \mu \mathrm{L}$ of a 1:100 dilution of each overnight grown bacterial culture in trypticase soy broth (TSB) (Merck, Germany) was inoculated into four wells of a 96-well flat bottomed polystyrene plate (Greiner Bio-One Inc., Germany). Following incubation at $37^{\circ} \mathrm{C}$ for $22-24$ hours, the cultures were removed and the wells were washed twice with $200 \mu \mathrm{L}$ of phosphate buffered saline ( $\mathrm{PBS}, \mathrm{pH}=7.4$ ) and dried at room temperature. Biofilms were stained with $0.1 \%$ safranin (Merck, Germany) solution in water for 15 minutes and the plates were washed in distilled water and dried at room temperature. The optical density (OD) of the biofilms was measured at $492 \mathrm{~nm}$ using an ELISA reader (Stat Fax 2100, Awareness Tech Inc., USA). Biofilm formation was considered negative at ODs below 0.12 , weakly positive at ODs 0.12-0.24 and strong positive at ODs > 0.24 (18). Each test was repeated on three different days and the results were reported as the mean of the obtained values.

\subsection{Detection of the pslA Gene}

Genomic DNA was extracted by boiling (19). PCR ampli- fication of the pslA gene was carried out using the following primers: pslA-F, 5'-CACTGGACGTCTACTCCGACGATAT-3'; pslA-R, 5'-GTTTCTTGATCTTGTGCAGGGTGTC-3' (Bioneer, Korea), generating an amplification product of $1119 \mathrm{bp}$ (20). The reaction mixture $(25 \mu \mathrm{L})$ contained $1 \mu \mathrm{L}$ of the extracted DNA, $1.5 \mathrm{mM} \mathrm{MgCl}_{2}, 0.4 \mathrm{mM}$ of each dNTP, $10 \mathrm{pM}$ of each primer and one unit of Taq DNA polymerase (CinnaGen, Iran). Amplifications were performed in a thermal cycler (Peqlab, Germany) using the following program: an initial incubation at $94^{\circ} \mathrm{C}$ for 10 minutes, followed by 30 cycles of one minute denaturation at $94^{\circ} \mathrm{C}, 30$ seconds annealing at $55^{\circ} \mathrm{C}$ and one minute extension at $72^{\circ} \mathrm{C}$ followed by 10 minutes at $72^{\circ} \mathrm{C}$. The amplification products were separated on $1 \%$ agarose gels, stained with Red Safe (Intronbio, Korea), and visualized using an image analysis system (UVItec, St John's Innovation Centre, UK).

\subsection{Metallo- $\beta$-Lactamase Detection}

MBL production was screened by the double-disc synergy test (17). Briefly, the turbidity of overnight grown bacteria in Mueller-Hinton (MH) broth (Merck, Germany) was adjusted to McFarland standard 0.5 before inoculating $\mathrm{MH}$ agar plates. An imipenem disc $(10 \mu \mathrm{g})$ was placed on the bacterial lawn $10 \mathrm{~mm}$ apart from a blank disc (edge to edge), to which, $10 \mu \mathrm{L}$ of an EDTA solution (0.5 M, pH: 8) was added before incubation at $37^{\circ} \mathrm{C}$ for 24 hours. Presence of an extended growth inhibition zone between the two discs was interpreted as positive for MBL production.

\subsection{Amp C Disc Test}

AmpC disc test was carried out as described previously (17). Briefly, a blank disc moistened with sterile saline was inoculated with a few colonies of the test strain. The disc was then placed next to a 30- $\mu$ g cefoxitin disc (Mast, UK) on the surface of an MH agar plate, previously inoculated with a lawn of Escherichia coli ATCC 25922 and incubated overnight at $37^{\circ} \mathrm{C}$. A flattening or an indentation of cefoxitin inhibition zone adjacent to the disc containing the test strain indicated AmpC $\beta$-lactamase production.

\subsection{Extended-Spectrum Beta-Lactamase Detection}

ESBL production was detected by the double-disc synergy test (DDST), as described before (17). Discs containing ceftazidime $(30 \mu \mathrm{g})$ and cefepime $(30 \mu \mathrm{g})$ were placed $15 \mathrm{~mm}$ apart from an amoxicillin/clavulanic acid disc (20 $+10 \mu \mathrm{g}$ ) on bacterial lawns before overnight incubation at $37^{\circ} \mathrm{C}$. ESBL production was detected when synergy was observed between the inhibition zones of cephalosporins and amoxicillin/clavulanic acid discs. Phenotypic confirmatory test for ESBL production was performed by placing a ceftazidime disc $(30 \mu \mathrm{g})$ alone and ceftazidime with clavulanic acid (10 $\mu \mathrm{g}$ ) on bacterial lawns before incubation at $37^{\circ} \mathrm{C}$ overnight. An increase of $5 \mathrm{~mm}$ in the inhibition zone around the combination disc was considered as ESBL production. 
Heydari S et al.

Figure 1. Amplification of the pslA gene in Pseudomonas aeruginosa burn isolates by polymerase chain reaction

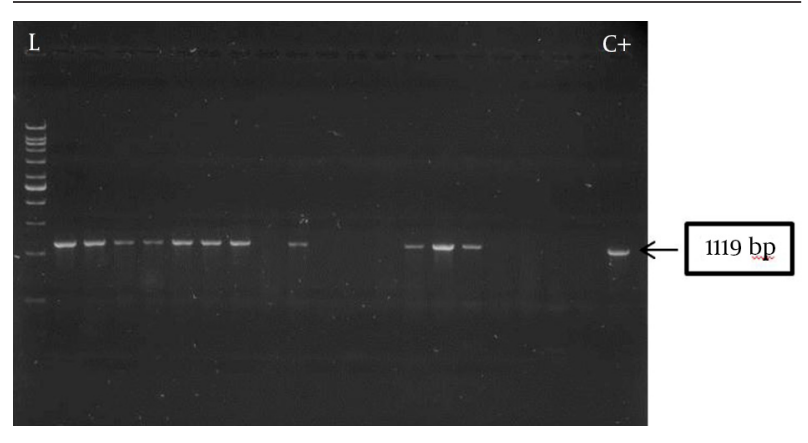

C+, control; L, 1 kb DNA ladder; lanes 2 to 20 , some test isolates.

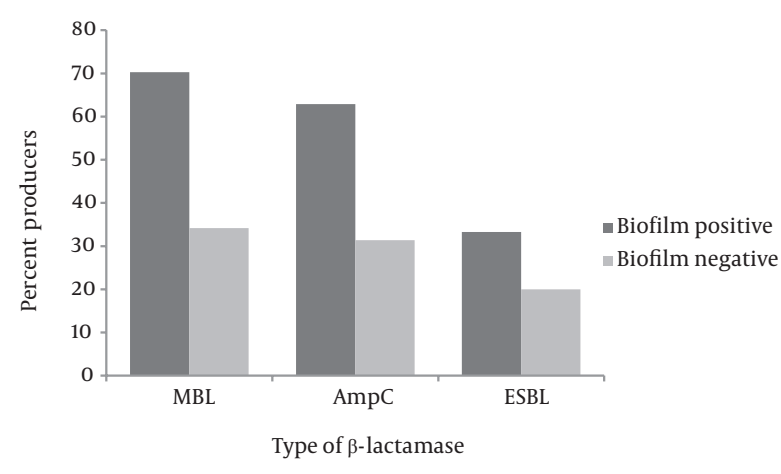

Figure 2. Production of MBL, AmpC and ESBL by biofilm-positive and biofilm-negative burn isolates of Pseudomonas aeruginosa

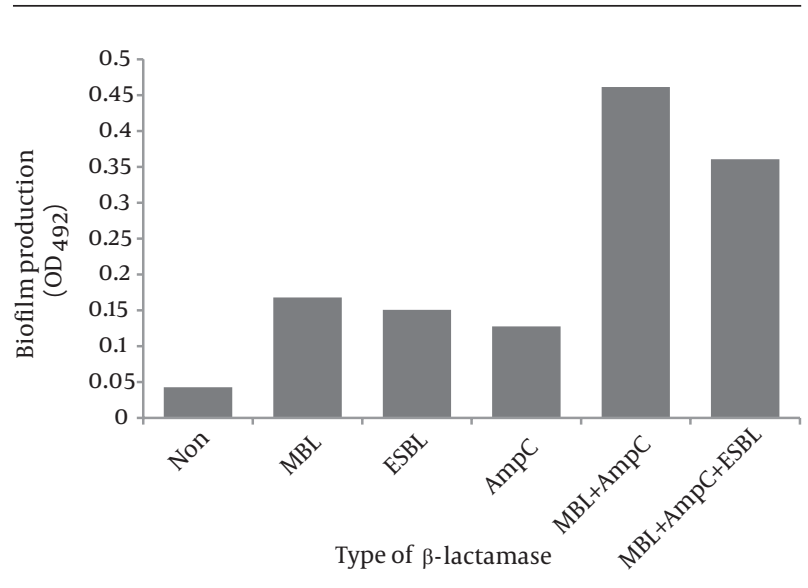

Figure 3. The Association between the degree of biofilm formation and $\beta$-lactamase production among burn isolates of Pseudomonas aeruginosa

\subsection{Data analysis}

Comparison of $\beta$-lactamase production between biofilm-negative and biofilm-forming isolates was carried out by the Mann-Whitney test, using SPSS V19.

\section{Results}

Of the 62 test isolates, 27 (43.5\%) formed biofilm, of which 18 (66.7\%) were strong and 9 (33.3\%) were weak producers with an average $\mathrm{OD}_{492}$ of $0.582 \pm 0.015$ and $0.194 \pm 0.001$, respectively. Presence of the pslA gene was observed in all biofilm producers, showing a strong association between pslA gene carriage and biofilm formation. Five biofilmnegative (14.2\%) isolates also carried the pslA gene. The amplification product of pslA gene for a number of $P$. aeruginosa burn isolates (1119 bps) is shown in Figure 1.

No significant difference was observed between the antibiotic susceptibility profiles of biofilm-positive and biofilm-negative isolates. Production of ESBL, MBL and AmpC $\beta$-lactamases by non-biofilm and biofilm producers is presented in Figure 2. Among the biofilm producing isolates, 19 (70.3\%) were MBL producers, 17 (62.9\%) produced AmpC, and 9 (33.3\%) were positive for ESBL production. Production of AmpC, MBL and ESBL in biofilm-negative isolates occurred in 12 (34.2\%), 11 (31.4\%) and 7 (20\%) isolates, respectively. As observed, MBL and AmpC productions were significantly higher in biofilm-positive strains $(\mathrm{P}=0.003$ and $\mathrm{P}=0.02$, respectively). Coproduction of AmpC and MBL was significantly higher in biofilm-positive isolates ( $n=12,44.4 \%)$ compared to biofilm negative ones $(n=7$, $20 \%)(P=0.00)$. Finally, three biofilm-positive $(11.1 \%)$ and one biofilm-negative isolates $(2.8 \%)$ produced all three $\beta$-lactamases. Phenotypic production of ESBL along with either AmpC or MBL was not detected.

The degree of biofilm formation in relation to $\beta$-lactamase(s) production is shown in Figure 3. As observed, strong biofilms were formed by the isolates that coproduced MBL and AmpC as well as the strains that had all three $\beta$-lactamases $\left(\mathrm{OD}_{492}: 0.46 \pm 0.020\right.$ and $0.36 \pm 0.060$, respectively). The isolates that produced one $\beta$-lactamase type formed weak biofilms (MBL producers $\mathrm{OD}_{492}: 0.17 \pm$ 0.015 , ESBL producers $\mathrm{OD}_{492}: 0.15 \pm 0.005$ and AmpC producers $\left.\mathrm{OD}_{492}: 0.13 \pm 0.012\right)$. Non- $\beta$-lactamase-producing strains did not form biofilms $\left(\mathrm{OD}_{492}: 0.043 \pm 0.001\right)$.

\section{Discussion}

$P$. aeruginosa is capable of causing chronic infections mostly due to its potential to form biofilms (16). The hallmarks of a mature biofilm include production of an extracellular matrix and increased resistance to antibiotics (11). In a recent study, 96\% of $P$. aeruginosa burn wound isolates were shown to form moderate to strong biofilms in vitro (21). Perez et al. showed the high potential of biofilm formation by clinical isolates of $P$. aeruginosa regardless of the specimen source (22). The association between the potential to form strong biofilms by P. aeruginosa and antibiotic resistance has also been shown (23). We found no significant difference between the antibiotic susceptibility profiles in biofilm-positive and biofilm-negative burn isolates of $P$. aeruginosa. In this research, $43.5 \%$ of our $P$. aeruginosa burn isolates formed biofilms, the majority of which (66.7\%) were strong biofilms. 
Presence of the pslA gene was shown as a good predictor of biofilm formation in non-mucoid isolates of $P$. aeruginosa in a number of studies $(13,15,20)$. We found that the pslA gene was present in all biofilm-producing $P$. aeruginosa burn isolates, suggesting a strong correlation between biofilm formation and pslA gene carriage. We also observed that $14.2 \%$ of the biofilm-negative isolates harbored the pslA gene. Hou et al. found that pslA gene was present in $31 \%$ of biofilm-negative ophthalmic P. aeruginosa isolates (20). This may suggests that gene presence does not necessarily result in its expression and biofilm formation is regulated by a complicated network of factors in addition to the pslA gene. The same observation has been made for biofilm formation in S. epidermidis (18).

Presence of different types of $\beta$-lactamases including AmpC, ESBL and MBL and the association of some of these enzymes with biofilm formation in P. aeruginosa has been shown in a number of studies $(5,24,25)$. In our study, MBL, AmpC and ESBL production occurred in 48.3\% (30/62), $46.7 \%(29 / 62)$ and $25.8 \%(16 / 62)$ of the isolates, respectively. However, MBL and AmpC production were significantly higher in biofilm-positive strains than biofilm-negative ones (70.3\% vs. $31.4 \%, 62.9 \%$ vs. $34.2 \%$, respectively). Coproduction of different $\beta$-lactamases has been observed by other investigators $(5,7,26)$. We detected MBL and AmpC coproduction in $30.6 \%$ of our isolates (19/62), the majority of which were biofilm-positive (63.1\%). Of the four isolates $(6.4 \%)$ that produced all three enzymes, 3 (75\%) were biofilm-positive.

Figure 3 shows the degree of biofilm formation in relation with $\beta$-lactamase production among our isolates. As observed, presence of two or three $\beta$-lactamases was associated with strong biofilms. Perez et al. showed that $P$. aeruginosa isolates harboring MBL gene produced strong to moderate biofilms in vitro (24). In another study, a highly significant association was found between the degree of biofilm formation and MBL production in P. aeruginosa (25). Similar results have been shown for Proteus mirabilis, where the potential to form biofilm was significantly higher in $\beta$-lactamase (AmpC and ESBL)-producing strains (27). However, another study showed that ESBL (but not MBL or AmpC) inhibited biofilm formation by impairing the twitching motility which plays an important role in micro-colony formation in P. aeruginosa (28).

In conclusion, biofilm formation correlated with pslA gene carriage as well as MBL and AmpC $\beta$-lactamase production in burn isolates of $P$. aeruginosa. More importantly, the isolates with multiple $\beta$-lactamase phenotypes produced strong biofilms in comparison to the strains with one type of $\beta$-lactamase that formed weak biofilms or $\beta$-lactamasenegative isolates that did not form biofilm at all.

\section{Acknowledgements}

The authors wish to thank the Shahid Beheshti University Research Council for providing a special grant to finance this research.

\section{Authors' Contributions}

Study concept and design: Fereshteh Eftekhar. Interpretation of data: Fereshteh Eftekhar, Samira Heydari. Drafting of the manuscript: Samira Heydari, Fereshteh Eftekhar. Critical revision of the manuscript for important intellectual content: Fereshteh Eftekhar. Statistical analysis: Samira Heydari. Study supervision: Fereshteh Eftekhar.

\section{Funding/Support}

Financial support for this research was provided by Shahid Beheshti University

\section{References}

1. Altoparlak U, Erol S, Akcay MN, Celebi F, Kadanali A. The time-related changes of antimicrobial resistance patterns and predominant bacterial profiles of burn wounds and body flora of burned patients. Burns. 2004;30(7):660-4.

2. Lipovy B, Rihova H, Hanslianova M, Gregorova N, Suchanek I, Brychta P. Prevalence and resistance of Pseudomonas aeruginosa in severely burned patients: a 10-year retrospective study. Acta Chir Plast. 2010;52(2-4):39-43.

3. Lambert PA. Mechanisms of antibiotic resistance in Pseudomonas aeruginosa. J R Soc Med. 2002;95 Suppl 41:22-6.

4. Mesaros N, Nordmann P, Plesiat P, Roussel-Delvallez M, Van Eldere J, Glupczynski Y, et al. Pseudomonas aeruginosa: resistance and therapeutic options at the turn of the new millennium. Clin Microbiol Infect. 2007;13(6):560-78.

5. Kumar V, Sen MR, Nigam C, Gahlot R, Kumari S. Burden of different beta-lactamase classes among clinical isolates of AmpC-producing Pseudomonas aeruginosa in burn patients: A prospective study. Indian J Crit Care Med. 2012;16(3):136-40.

6. Queenan AM, Shang W, Bush K, Flamm RK. Differential selection of single-step AmpC or efflux mutants of Pseudomonas aeruginosa by using cefepime, ceftazidime, or ceftobiprole. Antimicrob Agents Chemother. 2010;54(10):4092-7.

7. Upadhyay S, Sen MR, Bhattacharjee A. Presence of different betalactamase classes among clinical isolates of Pseudomonas aeruginosa expressing AmpC beta-lactamase enzyme. J Infect Dev Ctries. 2010;4(4):239-42.

8. Karatuna O, Yagci A. Analysis of quorum sensing-dependent virulence factor production and its relationship with antimicrobial susceptibility in Pseudomonas aeruginosa respiratory isolates. Clin Microbiol Infect. 2010;16(12):1770-5.

9. Costerton JW, Lewandowski Z, Caldwell DE, Korber DR, LappinScott HM. Microbial biofilms. Annu Rev Microbiol. 1995;49:711-45.

10. Mah TF, Pitts B, Pellock B, Walker GC, Stewart PS, O'Toole GA. A genetic basis for Pseudomonas aeruginosa biofilm antibiotic resistance. Nature. 2003;426(6964):306-10.

11. Wozniak DJ, Wyckoff TJ, Starkey M, Keyser R, Azadi P, O'Toole GA, et al. Alginate is not a significant component of the extracellular polysaccharide matrix of PA14 and PAO1 Pseudomonas aeruginosa biofilms. Proc Natl Acad Sci U S A. 2003;100(13):7907-12.

12. Ghafoor A, Hay ID, Rehm BH. Role of exopolysaccharides in Pseudomonas aeruginosa biofilm formation and architecture. Appl Environ Microbiol. 2011;77(15):5238-46.

13. Ma L, Jackson KD, Landry RM, Parsek MR, Wozniak DJ. Analysis of Pseudomonas aeruginosa conditional psl variants reveals roles for the psl polysaccharide in adhesion and maintaining biofilm structure postattachment. J Bacteriol. 2006;188(23):8213-21.

14. Jackson KD, Starkey M, Kremer S, Parsek MR, Wozniak DJ. Identification of psl, a locus encoding a potential exopolysaccharide that is essential for Pseudomonas aeruginosa PAO1 biofilm formation. J Bacteriol. 2004;186(14):4466-75.

15. Overhage J, Schemionek M, Webb JS, Rehm BH. Expression of the psl operon in Pseudomonas aeruginosa PAO1 biofilms: PslA performs an essential function in biofilm formation. Appl Environ Microbiol. 2005;71(8):4407-13. 
16. Matsukawa M, Greenberg EP. Putative exopolysaccharide synthesis genes influence Pseudomonas aeruginosa biofilm development. J Bacteriol. 2004;186(14):4449-56.

17. Salimi F, Eftekhar F. Coexistence of AmpC and Extended-Spectrum $\beta$-lactamases in Metallo- $\beta$-Lactamase Producing Pseudomonas aeruginosa Burn Isolates in Tehran. JundishapurJ Microbiol. 2013;8(6): e7178

18. Eftekhar F, Speert DP. Biofilm formation by persistent and non persistent isolates of Staphylococcus epidermidis from a neonatal intensive care unit. J Hosp Infect. 2009;71(2):112-6.

19. Sekiguchi J, Asagi T, Miyoshi-Akiyama T, Kasai A, Mizuguchi Y Araake M, et al. Outbreaks of multidrug-resistant Pseudomonas aeruginosa in community hospitals in Japan. J Clin Microbiol. 2007;45(3):979-89.

20. Hou W, Sun X, Wang Z, Zhang Y. Biofilm-forming capacity of Staphylococcus epidermidis, Staphylococcus aureus, and Pseudomonas aeruginosa from ocular infections. Invest Ophthalmol Vis Sci.2012;53(9):5624-31.

21. Jabalameli F, Mirsalehian A, Khoramian B, Aligholi M, Khoramrooz SS, Asadollahi P, et al. Evaluation of biofilm production and characterization of genes encoding type III secretion system among Pseudomonas aeruginosa isolated from burn patients. Burns. 2012;38(8):1192-7.

22. Perez LR, Costa MC, Freitas AL, Barth AL. Evaluation of biofilm production by Pseudomonas Aeruginosa isolates recovered from cystic fibrosis and non-cystic fibrosis patients. Braz J Microbiol. 2011;42(2):476-9.

23. Drenkard E, Ausubel FM. Pseudomonas biofilm formation and antibiotic resistance are linked to phenotypic variation. Nature. 2002;416(6882):740-3.

24. Perez LR, Antunes AL, Freitas AL, Barth AL. When the resistance gets clingy: Pseudomonas aeruginosa harboring metallo-betalactamase gene shows high ability to produce biofilm. Eur J Clin Microbiol Infect Dis. 2012;31(5):711-4.

25. Chakraborty D, Basu S, Chatterjee P, Dey SK, Das S. Concurrent determination of collagenase and biofilm formation activities in metallo-beta-lactamase producing Pseudomonas aeruginosa. Int J of Microbiol Res. 2011;2(3):208-12.

26. Oberoi L, Singh N, Sharma P, Aggarwal A. ESBL, MBL and Ampc beta Lactamases Producing Superbugs - Havoc in the Intensive Care Units of Punjab India. J Clin Diagn Res. 2013;7(1):70-3.

27. Nucleo E, Fugazza G, Migliavacca R, Spalla M, Comelli M, Pagani L, et al. Differences in biofilm formation and aggregative adherence between beta-lactam susceptible and beta-lactamases producing P. mirabilis clinical isolates. New Microbiol. 2010;33(1):37-45.

28. Gallant CV, Daniels C, Leung JM, Ghosh AS, Young KD, Kotra LP, et al. Common beta-lactamases inhibit bacterial biofilm formation. Mol Microbiol. 2005;58(4):1012-24. 